Extrême-Orient Extrême-Occident

\section{Extrême-Orient Extrême-Occident}

35 | 2013

Les astres et le destin. Astrologie et divination en Asie orientale

\title{
L'horoscope perdu des devins du Cambodge
}

The Lost Horoscope of Cambodia's Astrologers

François Bizot

\section{(2) OpenEdition}

Journals

\section{Édition électronique}

URL : http://journals.openedition.org/extremeorient/286

DOI : 10.4000/extremeorient.286

ISSN : 2108-7105

Éditeur

Presses universitaires de Vincennes

\section{Édition imprimée}

Date de publication : 1 mai 2013

Pagination : 171-197

ISBN : 978-2-84292-367-9

ISSN : 0754-5010

\section{Référence électronique}

François Bizot, "L'horoscope perdu des devins du Cambodge », Extrême-Orient Extrême-Occident [En ligne], 35 | 2013, mis en ligne le 01 mai 2016, consulté le 01 mai 2019. URL : http://

journals.openedition.org/extremeorient/286 ; DOI : 10.4000/extremeorient.286 


\title{
L'horoscope perdu des devins du Cambodge
}

\author{
François Bizot
}

En pays khmer, l'être voit le jour, existe et se meut dans un monde fermé, intérieur, «utérin»; c'est à la suite d'un regressus, d'un retour par les «états supramondains» (lokuttara = loka-udara), qu'il lui est donné de contempler les trois caractéristiques de l'existence, les quatre Chemins, les quatre Fruits, le Nibbāna ${ }^{1}$. Depuis fort longtemps les Hindus affirment que l'embryon a connaissance de l'acte bon et mauvais, contemple l'impérissable, pense à la syllabe $\mathrm{OM}$ et se souvient de sa naissance précédente.

Ainsi, les opinions ayant trait à l'au-delà, à la mort, à la vie de l'être transmigrant, sont communément théorisées, sinon sur la base d'une symbolique obstétrique nécessairement imagée et précise-comme c'est le cas par exemple pour les rites obligatoires de la vie ordinaire (naissance, puberté, ordination, mariage, funérailles) -, en se référant à l'idée d'une scène dramatique globale conçue comme une matrice. C'est par cet interstice spatial et temporel où se confondent secrètement les virtualités de la vie et les modalités latentes de tous les possibles, là où s'immisce l'existence embryonnaire, que se tisse, selon des signes établis par les dieux, la trame des destinées humaines. Sont alors signifiants toute une série d'indices et de calculs grâce auxquels la tradition peut produire des interprétations magiques, une fois ceux-ci déchiffrés à l'aide d'une grille, en les rapportant le plus souvent aux signes avant-coureurs d'un événement édifiant, dérivé d'un Jātaka ou tiré d'un récit légendaire devenu système divinatoire ${ }^{2}$.

Cette conception de la marche des choses peut aussi bien remonter au vieux fond des croyances prénatales de l'Asie du Sud-Est qu'aux conceptions indiennes de la cosmologie traditionnelle. Entre-temps, elle est devenue intrinsèque à l'enseignement du bouddhisme, de ses règles, de ses correspondances, et perdure

1. Bizot 1992 a: 46, 60 .

2. Leclère 1909, Porée-Maspéro 1962, Hang Thun Hak 1969, Wales 1983. 
en marge des nombreuses façons de croire qui relient entre eux une grande partie des habitants de la péninsule indochinoise ${ }^{3}$.

De ce fait, la divination est devenue un mode de connaissance complexe qui intervient à tous les niveaux; sa fonction, ses procédés ne sont pas ceux d'un domaine de la pensée isolé et circonscrit, susceptible d'être extrapolé. Au contraire, il faut aborder les savoirs divinatoires comme le lieu privilégié des interactions qui existent - à la fois conceptuellement et matériellement - entre les domaines de la religion, du rituel, du culte des morts, des rites de guérison, etc. ${ }^{4}$. Les matériaux offerts par les pays de l'Asie du Sud-Est sont foisonnants à cet égard, aussi bien du point de vue des ressources textuelles que des pratiques. Non seulement astrologues, oracles et devins font partie de la collectivité des maîtres que consulte régulièrement l'ensemble des populations, mais leur savoir est utilisé par les moines au monastère, ainsi que par les spécialistes du culte - ācāry (ācariya), grū (guru), hora, guérisseurs, médiums, géomanciens - au village.

À cet égard le royaume est perçu comme un cœur vivant en même temps qu'un lieu sacré où le mont Meru se dresse au centre du zodiaque. C'est une allégorie du corps rénové de l'adepte, celui que le yogin (i.e. le Yogāvacar) a pour mission d'intégrer au cours de ses exercices, avec sa géographie, ses routes, ses points cardinaux, ses montagnes, ses étages célestes, ses planètes, ses dieux, ses démons, ses périls et ses refuges, à l'image de cette matrice où croît le «Figuier à cinq branches » ${ }^{5}$. Le monde n'est jamais qu'un vaste amphithéâtre; le combat des forces du bien et du mal s'y étage, et son influence sur l'existence des êtres vivants s'y exerce par le biais des planètes avec lesquelles ces forces sont en relation de correspondance. Plus précisément, les devins du Cambodge ont colporté cette science en y rapportant de nombreux épisodes archétypes, tirés du Rāmāyaṇa indien, parce que les héros de la célèbre épopée, à la suite d'un transfert entre leurs exploits et les choses, sont sensés influer sur le destin des hommes.

Il faut savoir en effet que toute l'activité de l'adepte est tendue vers l'Éveil d'un «Joyau» ou «Globe de cristal» dont le siège se trouve dans l'ombilic. Il est précisé qu'au cours des exercices d'ascèse, l'esprit du Yogāvacar s'éprend du Joyau situé dans le vide au milieu du nombril et que le Joyau n'est autre que le Dhamma ou le Bouddha. Dans le Rāmaker (Rmk) - p.rāmaker (ti) «la gloire de Rām», du nom donné au Cambodge et en Thaillande ${ }^{6}$ à la version locale du

\footnotetext{
3. Bizot 1992 a, 1993, 1998, 2000.

4. Guenzi 2010.

5. Bizot 1976 .

6. Bizot 1989: 33-35. Au Siam, les Thaïs connaissent la même version sous le nom de Rāmakīer, Nicolas 1928.
} 
Rāmāyaṇa, cet élan du fidèle est métaphorisé par la conquête amoureuse de Nān Setā.

La version complète du Rāmaker éditée au Cambodge et les études associées consacrées à ses sources et à sa dimension mystique ${ }^{7}$, montrent que la structure de l'œuvre, paradoxalement caractérisée par des interpolations originales, est une mise en scène de l'adepte bouddhiste dans son cheminement vers l'immortalité. Il ne s'agit peut-être pas à proprement dire d'une version «ésotérique » - la pensée symbolisée n'y est pas exprimée -, mais plutôt d'une lecture spéciale, d'une «appropriation» du vieux poème épique, modifié selon la perspective philosophique et didactique que prêtent les Cambodgiens à l'effort ascétique. Tout est mis en place autour d'une idée centrale et de son corrolaire: la quête de Nān Setā, symbole de l'achèvement spirituel, et l'ascèse mise en œuvre pour y parvenir. Principe immatériel mais indestructible, la belle Setā (p. seta «blanc», «pur») symbolise le véhicule d'immortalité, et l'on peut considérer que cela s'apparente aux croyances en un «soi» transmigrant. Pour être plus précis, si le «Joyau» est bien au singulier une sorte d'équivalent de l'ātman éternel et lumineux, au pluriel, les «Joyaux» sont les caractères de l'immortalité qui s'acquièrent virtuellement par l'initiation ${ }^{8}$. Ces caractères sont ceux d'un nouveau «corps» $(k \bar{a} y a)$, élaboré à l'aide des lettres de la «doctrine» (dhamma); l'aboutissement en est le «Joyau », c'est-à-dire, pour employer un terme de la philosophie bouddhique: le «dhammakāya ${ }^{9}$. Cette idée résume à elle seule l'Enseignement tel qu'il a été interprété chez les Khmers. C'est à partir de cela que s'est développée toute une symbolique relative aux initiations, et que l'on retrouve dans la divination. Ainsi, la formation de l'individu s'élaborant dans un ventre maternel, il n'est pas surprenant que s'y produisent également les conditions de son devenir ici-bas.

En pratique, il s'agit de maîtriser le processus par le façonnement d'un corps immaculé, le degré de pureté obtenu conditionnant la renaissance. S'il est entièrement débarrassé des souillures qui lient à l'existence, il conduit le disciple au Nibbāna; s'il ne l'est que partiellement, celui-ci retourne à la vie mortelle, mais dans un état plus avancé sur la voie, ne comportant plus qu'un nombre limité de renaissances. Le devin représente Māhā Paț̣ān, le dieu instructeur qui est indispensable au voyage de l'être transmigrant. Dans le Rāmaker, celui-ci est personnifié par Bibhek, dont l'apport stratégique permet toujours à Brah Rām d'affronter victorieusement les épreuves faisant obstacle à son union avec

7. Martini 1978, Bizot 1973, 1981, 1989.

8. Bizot 1989: 20-25.

9. Bizot 1992b. 
Nān Setā $\bar{a}^{10}$. Sous cet angle la divination n'est rien d'autre qu'une initiation, en d'autres termes une méthode d'investigation. De même que le succès de l'effort ascétique repose sur l'importance du nissaya de chacun, c'est-à-dire des «protections», des «supports », des «aptitudes requises», qui résultent de la plus ou moins grande accumulation de mérites au cours des existences passées; de même, ici-bas, l'oracle est rendu par l'examen des forces mises en présence au moment de la naissance. Le devin raccroche le devenir du sujet à la biographie des héros de l'épopée; le Rāmaker devient ici l'histoire de tout le monde.

Ce court article n'a pas l'ambition de poser, même très succinctement, les jalons d'une ethnographie de la divination au Cambodge, et encore moins de relever les questionnements que ce champ d'étude peut soulever par rapport aux théorisations de l'anthropologie. En m'appuyant sur les textes et les matériaux ethnographiques que j'ai pu recueillir sur place (de 1965 à 2011), mon intention est de décrypter cette pratique divinatoire qui consiste à mettre en scène des personnages de l'histoire de Rām et de Setā. Ce faisant, contrairement à ce qu'on pouvait attendre, je montre que le système des signes et des aphorismes utilisés ne restituait pas la version adaptée au modèle cambodgien, mais que, le plus fidèlement possible, et probablement depuis Angkor, les devins s'étaient attachés à transmettre le poème tel qu'ils l'avaient reçu, en partant de sa composition primitive.

Les Cambodgiens ont un calendrier luni-solaire, à la fois basé sur le mouvement de la lune et corrigé en accord avec l'année solaire, mais ils ne tiennent compte couramment que des lunaisons sur lesquelles se fonde le calendrier religieux. Celles-ci portent des noms d'origine sanskrite, et sont désignées par un chiffre qui fait commencer l'année au mois de maksir (novembre/décembre). Les mois sont divisés en deux parties: la première, dite «croissante» (khnöt), va de la nouvelle lune à la pleine lune; et l'autre, dite «décroissante» (rnoc), part du lendemain de la pleine lune et comporte quatorze ou quinze jours. Enfin, à côté de la chronographie indienne, les Cambodgiens se servent aussi, depuis au moins la fin du $\mathrm{x}^{\mathrm{e}}$ siècle, du cycle chinois des douze animaux se succédant indéfiniment pour désigner l'année. Ce cycle, combiné avec divers numéros d'ordre, forme un comput de soixante années inconnu en Inde. Dans ce système, la nouvelle année qui marque les changements de nom et de quantième débute le premier jour de cet (mars/avril).

Toutes les populations de la péninsule indochinoise connaissent un cycle duodénaire comparable, identique au système chinois. Curieusement, dès le $\mathrm{XIV}^{\mathrm{e}}$ siècle, les Siamois donnent aux douze animaux des noms qu'on retrouve aussi en khmer, mais qui sont apparentés à des formes très anciennes, communes

10. Bizot 1989: 51, 57. 
aux langues muong et vietnamienne ${ }^{11}$. Les autres peuples de langue taï (Lao, Ahom, Shan, etc.) comptent les jours, les mois et les ans, au moyen d'un cycle sexagénaire formé par la combinaison d'une série duodénaire avec une série dénaire ${ }^{12}$.

Ces animaux ont partout une grande importance. C'est suivant l'animal présidant à l'année de naissance que l'on règle les questions qui se posent, telles que le traitement à suivre ou les sacrifices à faire pour guérir un malade, fixer les prières pour conjurer un malheur, déterminer le moment propice d'entrer dans une maison neuve, etc., et que l'on tire les horoscopes qui déterminent la possibilité ou l'impossibilité, par exemple, d'entreprendre un voyage, de contracter un mariage, etc.

L'origine du cycle des douze animaux remonte au mythe de la création. Au commencement de l'humanité, la première femme prend naissance à partir de l'élément terre, et son époux, à partir de l'élément feu. Tous deux mettent au monde un garçon, une fille et un hermaphrodite, puis créent le Mont Meru, le soleil, la lune, etc. Leur progéniture donne naissance à treize enfants (dont l'un meurt) qui reçoivent dans l'année de leur naissance des jouets modelés en forme d'animaux. Ces figurines prirent vie et devinrent les ancêtres des espèces actuelles. Chaque enfant fit reposer sa santé sur cet animal et pour guérir il fallait relâcher celui correspondant à sa naissance, lequel devenait son génie protecteur. L'image gardée sur soi était le gage de sa prospérité. Le nom des animaux servit à nommer les années du cycle duadécimal: année du rat, du bœuf, du tigre, du lièvre, du grand serpent (ou dragon), du serpent, du cheval, de la chèvre, du singe, du coq, du chien, du porc (ou éléphant) ${ }^{13}$.

D'autre part, les Khmers font usage d'un système basé sur le Rāmāyaṇa, sans intervention de la position astronomique des corps célestes pris seulement comme symboles numériques. Ils recourent pour cela à des calculs et à des tables de correspondances consignés dans les traités d'astrologie, sans égard au calendrier sexagésimal, parce que ce système fonctionnait déjà par lui-même au début, indépendamment du cycle des douze animaux ${ }^{14}$. Les augures sont

11. Cœdès $1935: 316$.

12. Finot 1917: 76-83, Billard 1963: 404.

13. Bizot 1996: 54-55, 65, 231-232.

14. On trouve bien des listes où sont mis en correspondance les animaux du cycle avec les protagonistes de l'épopée indienne: 1 . années du rat/race des dieux/siège Bibhek; 2. année du bœuf/race des hommes/siège Brah Rām; 3. année du tigre/race des yakhs/ siège Khar ; 4. année du lièvre/race des hommes/siège Brah Lakh; 5. année du dragon/ race des dieux/siège Nān Setā (ou Sedā, ou Sītā); 6. année du serpent/race des hommes/ siège Hanumān; 7. année du cheval/race des dieux/siège Rāp; 8. année de la chèvre/ race des dieux/siège Bibhek; 9. année du singe/race des yakhs/siège Brạ̣ Rām; 10. 
tirés d'après l'arrangement d'épisodes se rapportant à des personnages types : Braḥ Rām (Rāma), Nān Setā (Sītā), Rāb (Rāvaṇa), Bibhek (VibhīÒạ̣a), Khar/ Dūbhī (Ghara ou Khāra), Braḥ Lakh (Lakṣmāṇa), Hanumān (Hanumat), Setayūs (Jețāyus). Suivant l'année de sa naissance, le sujet reçoit pour «siège» un de ces huit personnages (dinamin $)$ et dépend de la «race» des hommes, des démons (yakhs) ou des dieux. C'est ainsi, par exemple, que des jeunes gens ayant pour «véhicule» respectif Rāp et Nān Setā, ou appartenant à la race des démons et à la race des dieux, ne peuvent se marier sans danger.

Parmi les manuscrits qui ont été sauvés du Cambodge en 1975 dans le cadre de mes «Recherches sur le bouddhisme khmer», et en particulier ceux rédigés sur «papier indigène» $(k r a \bar{m} m \dot{n})$, certains font de longues mentions aux pratiques divinatoires. Ces manuels, jamais datés, souvent illustrés, sont à l'usage des maîtres et par voie de conséquence offrent peu d'explications. Leur objet est de fixer par écrit les prescriptions et formules nécessaires à l'expression de l'oracle et de servir de support aux calculs. Si leur rédaction peut remonter au $\mathrm{XVIII}^{\mathrm{e}}$ siècle pour certains (la durée de ce type de support très épais peut dépasser plusieurs siècles), il s'agit de copies dont le contenu est généralement beaucoup plus ancien.

Cinq manuscrits ont été consultés (TK 280, TK 287, TK 300, TK 307, TK 480), dont trois en particulier (TK 287, TK 307, TK 480), parce qu'ils exposent, sous forme de sentences extraites des épisodes d'un Rāmāyaṇa, deux façons différentes de réaliser les prédictions. La première est intitulée les «Huit influences », la seconde les «Flèches de Braḥ Rām».

\section{Les huit influences}

Cette méthode implique l'usage de deux «grilles » (târāi்) de chacune 32 cases réparties selon quatre colonnes de 8 chiffres (Fig. 1 et 2). Les colonnes correspondent, de gauche à droite, aux âges de la vie: enfance, adolescence, maturité, vieillesse, des héros de l'épopée. Les chiffres sont associés aux «huit influences » (athagroh) ou «planètes » (tons $\bar{a})$ : les sept jours de la semaine, plus Rāhū intercalé entre le jeudi et le vendredi. Dimanche a le nombre 1, lundi le nombre 2 , mardi le nombre 3 , mercredi le nombre 4 , jeudi le nombre 5 , vendredi le nombre 6, samedi le nombre 7, Rāhū le nombre 8. La numérotation est donnée

année du coq/race des yakhs siège Rāp; 11. année du chien/race des yakhs/siège Brạ̣ Lakh; 12. année du porc/race des hommes/siège Nān Setā. De telles tables sont toutefois incomplètes, dispersées, difficiles à utiliser, évidemment plus récentes et en tout cas postérieures à l'introduction des premières versions du Rāmāyaṇa à Angkor, mais au final assez peu employées par les devins eux-mêmes. $C f$. Porée-Maspero 1962: 320. 
dans un ordre particulier et différent suivant que le sujet est une femme ou un homme. Pour une femme: 1, 2, 3, 4, 5, 8, 7, 6 (Fig. 1); pour un homme: 7, 5, 8, 6, 1, 2, 3, 4 (Fig. 2).

\begin{tabular}{|l|l|l|l|}
9 \\
\hline
\end{tabular}

Fig. I

\begin{tabular}{|l|l|l|l|}
\hline 7 & 7 & 7 & 7 \\
\hline 5 & 5 & 5 & 5 \\
\hline 8 & 8 & 8 & 8 \\
\hline 6 & 6 & 6 & 6 \\
\hline I & I & I & I \\
\hline 2 & 2 & 2 & 2 \\
\hline 3 & 3 & 3 & 3 \\
\hline 4 & 4 & 4 & 4 \\
\hline
\end{tabular}

Fig. 2

On compte, de haut en bas et de gauche à droite, autant de cases que le sujet a d'années, revenant à la case départ tous les 32 ans. Le chiffre obtenu permet de déterminer, à l'aide d'une liste de concordances, le point cardinal imputé : 1 $=$ Nord-Est, $2=$ Est, $3=$ Sud-Est, $4=$ Sud, $7=$ Sud-Ouest, $5=$ Ouest, $8=$ NordOuest, $6=$ Nord.

Enfin, ce point cardinal renvoie à un personnage du Rāmāyaṇa:

Nord-Est: Bibhek

Sud-Ouest: Rāb

Est:

Brah Rām

Ouest:

Nān Setā

Sud-Est: Khar/Dūbhī

Nord-Ouest: Setayūs

Sud:

Brạ̣ Lāks

Nord:

Hanumān

Pour chaque personnage sont donnés quatre épisodes. Le devin retient celui qui correspond à la colonne enfance, adolescence, maturité ou vieillesse, en fonction du chiffre obtenu. Par exemple, le chiffre d'une femme de 40 ans sera le « 6 » dans la colonne «enfance»; celui d'un homme de 25 ans sera le «7» dans la colonne «vieillesse». Le «siège» (dinamin) pour la femme de 40 ans sera donc «Hanumān enfant» et celui de l'homme de 25 ans «Rāb vieillard».

Voici la liste des épisodes tels qu'ils sont donnés dans TK $480^{15}$ :

15. Pour la translittération du Cambodgien, $c f$. Tableau des signes diacritiques, in: Bizot 1996. 


\section{Bibhek}

1. [A24] siddhikāryy doh dinamin nūv bīybhek kmīǹ pān jā apparājjā ammcāss khluon è āsraiy tèh khluon è doh thkāt mān thmah pāk bvam noh sammröt nūv phdah è aoy dos trūv khmoc ābuk èn aoy sūn rūp prus 1 nān 3 beh rūtth 3 kandon 5 jūn dov ìsān thñaiy 3 saḥ hón

«Succès! Si le siège est en Bibhek enfant: "Il est vice-roi" [Rmk?] ${ }^{16}$. Chacun ne peut s'en remettre qu'à soi-même. Si l'on est malade, la faute en est une pierre ou du bronze cassé enfoui sous la maison; le $\operatorname{khmoc}^{17}$ du père nous a frappé. Modeler 1 image d'homme et 3 de jeune fille dans un bè ${ }^{18}$ à 3 ruot [contenant] 5 kandon ${ }^{19}$ et le déposer au Nord-Est. Guérison dans les trois jours.»

2. doh dinamin nūv bīybhek bāl dāy thā rābbh paṃmpar'pan் doḥ dinaṃn nūv nèh nūv köt put cammnèh èn nūv sabv thriaiy jā dukkh doḥ thkāt krèn pān் ph-Un è khön phan gnāh doh thkāt jammmị̣̄h crön jhịh kpāl nimit sasar'drut bvam noh jön klīy aoy doss pröh sūn rūp braḥ rāmm 1 rūp devatā jīh tamrīy 1 beh rūtth 6 jūn dov Isān thriaiy 3 saḥ hòn

«Si le siège est en Bibhek adolescent: "Il prédit, et Rāb le bannit" [Rmk 23]. Si le siège est celui-là, [le danger] naît de notre propre science. Aujourd'hui le malheur s'est abattu sur nous. Si l'on est malade, on peut craindre que ce soit à cause de la famille; si l'on souffre notamment de maux de tête, un pilier [de la maison] s'est creusé, sinon il est trop court. Modeler 1 image de Brạ̣ Rām, 1 image d'un dieu sur un éléphant dans un bè à 6 ruot et le déposer au Nord-Est. Guérison dans les 3 jours.»

3. doh dinamin nūv bīybhek kanțāl aoy prayāt krèn mān kț̄yy smöh slāpp khlūn $n \bar{u} v$ brāt braḥbandh kūn cov è nūv sabv thriaiy jā dukkh nūv It pramān pān löy gey möl i்ñāy doh thkāt trūv khmoc ābuk è khön phan aoy prayāt bhlön nūv chèh phdah aoy sūn rūp satv 1 sūn rūp khlāh 1 rūpp pana'kkhāh 1 rūp braḥ rām debv 1 brah

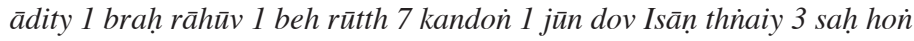

«Si le siège est en Bibhek adulte: faire attention. Craindre la survenue d'un conflit mortel ou d'une séparation de l'épouse et des enfants [Rmk 23]. Aujourd'hui un malheur sans fin s'est abattu sur nous. On nous méprise. Si l'on est malade, le khmoc du père s'est fâché contre nous. Prendre garde qu'un incendie n'éclate dans la maison. Modeler 1 image d'animal, modeler 1 image de tigre, 1 image de Puññakāy, 1 image de Braḥ Rām, 1 du soleil, 1 de Rāhū, dans un bè à 7 ruot [contenant] un kandon et le déposer au Nord-Est. Guérison dans les 3 jours.»

16. Je donne ici entre crochets le numéro du passage en référence dans le Rāmaker; $c f$. traduction française in: Bizot 1989.

17. khmoc: le fantôme, l'esprit d'un mort.

18. bè: il s'agit d'un plateau fabriqué à l'aide d'une lanière de tronc de bananier maintenue par des baguettes de bambou; la lanière est arrangée de façon à revenir sur elle-même en un nombre rituel de «tours» ou ruot. Les figurines sont modelées à l'aide d'argile ou de farine.

19. Le kandon est un petit réceptacle en feuille de bananier destiné à recevoir de la nourriture, des chiques de bétel, des cigarettes, etc. 
4. [A25] doḥ dinamin nūv bīybhek cāss brah rām aoy svoy rāj sammpātti nūv

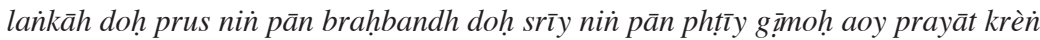
pañ ātmāh bvam noḥ khñamm ratth doḥ thkät brah lịn min nūv knan் khluon löy aoy pūjā brah groh höy aoy dadūl brah lịn aoy sūn rūp brah paramīysūr 1 nā̀n bhogavatīy 1 yaks kān tammpon் 1 beh rūtth 4 kandon் 7 jūn dov Isān thinaiy 3 sah hon

«Si le siège est en Bibhek vieillard: "Brah Rām le fait régner à Lañkā" [Rmk 66]. S'il s'agit d'un homme, il va trouver une épouse; s'il s'agit d'une femme, elle va trouver un mari. Sinon la survenue d'un accident ou une obligation de s'enfuir est à craindre. Si l'on est malade, les esprits vitaux ont quitté notre corps. Exorciser le mal et faire revenir les esprits vitaux. Modeler 1 image d'Íśvara, 1 de Bhogavatī 1 d'un yaks tenant un bâton, [dans] 1 bè à 4 ruot [contenant] 7 kandon் et le déposer au Nord-Est. Guérison dans les 3 jours.»

\section{Braḥ Rām}

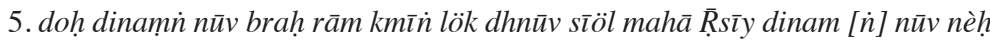
pān satv catupād dvepād anak ddhamm nūv aoy broh yak āsā gey doḥ prus nin pān brạ̣bundh doḥ srīy nin pān phțīy bvam noh nin pān khñamm doḥ thkāt trūv khmoc ābuk èn pröh sūn rūp prus 1 nā̀n 1 brāy 2 sèh 2 beh rūtth 3 kandon் 7 jūn dov pūr thriaiy 3 sah hón

«Si le siège est en Braḥ Rām enfant: "Il soulève l' arc magique du Grand-ascète" [Rmk 14]. Si le siège est celui-là: acquisition d'animaux quadrupèdes ou bipèdes due à une personne importante que nous servons. Un homme va rencontrer une épouse, une femme un mari, sinon des serviteurs. Si l'on est malade, le khmoc du père a frappé. Il convient de modeler 1 image de garçon, 1 image de fille, 2 images de $b r a \bar{a} y^{20}, 2$ images de chevaux [dans] un pè à 3 ruot [contenant] 7 kandoñ et le déposer à l'Est. Guérison dans les 3 jours.»

6. doh dinamin nūv brah rām bāl pān setā höy rābbh lūc vin doh tör aoy prayāt

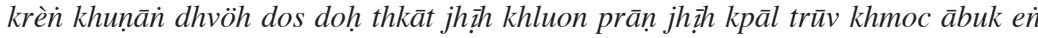
aoy sūn rūp prus 5 srīy 5 beh 7 rūtth chāt dun 10 kandon 18 jūn dov [A26] pur thriaiy 3 sah hò

«Si le siège est en Braḥ Rām adolescent: "Il a obtenu Setā, mais Rāb la ravit" [Rmk 18]. Si l'on doit aller quelque part, faire attention qu'une Khun Nān ne vienne nous porter malheur. Si l'on est malade, qu'on souffre de partout, qu'on a mal à la tête, le khmoc du père nous a frappé. Modeler 5 images de garçon, 5 de fille [dans] un bè à 7 ruot [contenant] 10 fanions à étages et 18 kandon், et le déposer à l'Est. Guérison dans les 3 jours.»

7. doḥ dinaṃn் nūv brạ̣ rām kanịāl tām pröss mās rābbh lūc nāàn setā E kroy aoy prayāt satv jön 24 aoy dos doh thkät aoy sūn rūp brāy 14 pruss 1 beh rutth 5 kandon் 7 jūn dov pūr thriaiy 3 sah hon

«Si le siège est en Brah Rām adulte: "Il poursuit la gazelle d'or. Pendant ce temps, Rāb enlève Nān Setā” [Rmk 18]. Faire attention que des animaux bipèdes

20. brāy: âme errante. 
ou quadrupèdes ne nous portent malheur. Si l'on est malade, modeler 14 images de brāa, 1 de garçon [dans] un bè à 5 ruot [contenant] 7 kandon et le déposer à l'Est. Guérison dans les 3 jours.»

8. doḥ dinaṃn் nūv braḥ rām cāss pān sammpātti laìkāh dinaṃn் nūv nèh pān satv catupād dhvehpād anak phan் sraleñ doḥ tör mān tammkāt put sasar'phdah drut bvam noḥ jön klīy bvam noḥ bhüm dābv aoy dos bvam noḥ khmoc yak braḥ lịn dov duk knai் gohā ddhammm aoy pūjā brah groḥ dadūl brah lịn höy aoy sūn rūp brāy 4 beh rūtth 6 pambun tāk srāh 1 jūn dov pūr thinaiy 3 sah hon

«Si le siège est en Brạ̣ Rām vieillard: "Il obtient les richesses de Lan̉kā" [Rmk?]. Si le siège est celui-là: acquisition d'animaux quadrupèdes ou bipèdes ; des gens nous veulent du bien. Si l'on doit se déplacer quelque part, il y a danger: un pilier de la maison est creux ou trop court; sinon, c'est le sol en pente qui porte malheur; sinon, un khmoc va se saisir de nos esprits vitaux pour les enfouir dans une grotte profonde. Exorciser le mal et faire revenir les esprits vitaux. Modeler 4 images de brāy [dans] un bè à 6 ruot [contenant] un tube de bambou plein d'alcool, et le déposer à l'Est. Guérison dans les 3 jours.»

\section{$\operatorname{Ghar}^{21}$}

9. doh dinaṃn் nūv ghar kmīn Dūrabhñ̄y ūt prayut nin ābuk en mān kțīy aoy prayāt citr En் doḥ thkāt aoy pūjā brah groḥ aoy sūn rūp stec 1 muntrīy 4 beh rūtth 3 kandon் 4 jün dov agneh thriaiy 3 sah hon

«Si le siège est en Ghar enfant: "Dūbhī affronte orgueilleusement son propre père" [Rmk 20]. En cas de conflit, se méfier de soi-même. Si l'on est malade, exorciser le mal. Modeler 1 image de Prince, 4 de dignitaires [dans] un bè à 3 ruot [contenant] 4 kandon et le déposer au Sud-Est. Guérison dans les 3 jours.»

10. doh dinaṃn nūv ghar bāl dūrabhīy pān sammpātti ābuk è nūv mān lābbh catu pād dvepād bvam noḥ aoy prayāt krèn bhlön chèh sammbat kum prahèss löy gey nūv dhvö dos [A27] kum duk citr gey begy löy doh thkāt putth chīy sec aoy sūn rūp brāy 6 beh rütth 4 kandon் 4 jūn dov agneh thriaiy 3 sah hon

«Si le siège est en Ghar adolescent: "Dūbhī obtient les richesses de son père" [Rmk 20]. Chance! [Acquisition] d'animaux quadrupèdes ou bipèdes. Sinon, attention: craindre que du feu enflamme des étoffes. Ne pas être distrait. Quelqu'un nous veut du mal. Ne pas faire confiance trop facilement aux gens. Si l'on est malade, c'est qu'on a mangé de la viande. Modeler 6 image de brāy [dans] un bè à 4 ruot [contenant] 4 kandon et le déposer au Sud-Est. Guérison dans les 3 jours.»

21. Dans le Rāmaker, comme dans le Rāmakīen, en thaï, il existe un Yaks nommé Khar, neveux de Rāb (Rmk 12). En revanche, «Ghar» est inconnu avec cette orthographe. Il s'agit peut-être d'un équivalent pour Dūbhī, puisqu'il est dit combattre Bālī. Mais cela n'explique pas que les deux noms soient employés simultanément. En tout cas, les présages sont tirés des aventures de Dūbhī et non pas de Khar ou Ghar. 


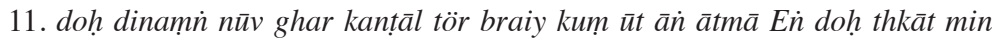
sranuk citr Ė̉ mān tammmūk aoy dos aoy sūn rūp khlā 3 kandon 7 jūn dov agneh thriaiy 3 sah hò

«Si le siège est en Ghar adulte: "Il marche en forêt" [Rmk 20]. Ne pas se vanter. Si l'on est malade ou qu'on éprouve de l'inquiétude, une termitière provoque le mal. Modeler 3 images de tigre [dans un bè contenant] 7 kandon et le déposer au Sud-Est. Guérison dans les 3 jours.»

12. doh dinamin nūv ghar cāss prayut nin bālīy gey sap khluon sabv thnaiy jā dukkh doḥ thkāt trūv khmoc ābuk En bvam noh bhūm kbuss aoy dos aoy sūn rūp bālīy sbāy krapīy 1 brāy 6 beh rūtth 4 kandon் 8 jūn dov agneh thriaiy 3 sah hon

«Si le siège est en Ghar vieillard: "Il se bat avec Bālî" [Rmk 20]. Quelqu'un nous déteste. Aujourd'hui on est malheureux. Si l'on est malade, le khmoc du père nous a frappé. Sinon, c'est une surélévation du sol qui porte malheur. Modeler 1 image de Bālī portant le buffle sur son épaule, 6 brāy [dans] un bè à 4 rūtth [contenant] 8 kandon et le déposer au Sud-Est. Guérison dans les 3 jours.»

\section{Brah Laks}

13. doḥ dinaṃin nūv brạ̣ lākkh kmìn dov möl setā knà் gohā dinaṃn nūv nèh krèn pon் ph-Un pracand phan் gnāh doh thkāt trūv khmoc tāy hon aoy dhvöh beh rūtth 4 kandon் 8 jūn dov dāksīön thriaiy 3 sạ̣ hòn

«Si le siège est en Brạ Laks enfant: "Il va voir Setā dans la grotte" [Rmk?]. Si le siège est celui-là: crainte de jalousies dans la famille. Si l'on est malade, le khmoc [d'une personne disparue] de mort violente nous a frappé. Faire un bè à 4 ruot [contenant] 8 kandon et le déposer au Sud. Guérison dans les 3 jours ».

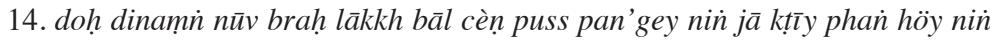
mān lābbh catupād dvepād doh thkāt brah lịn min nūv knaì khlūn trūv khmoc ābuk En் yak brāy 7 anak mak dhvöh dos put bhüm gey kapp khmoc brāy aoy sūn rūp brah lāk 1 [A28] beh rūtth 4 kandon 6 jūn dov dāksīön thriaiy 3 sah hon

«Si le siège est en Brah Laks adolescent: "Il s'en va pratiquer les observances" [Rmk 12]. Une personne va nous préserver d'un conflit. Chance! [Acquisition] d'animaux quadrupèdes ou bipèdes. Si l'on est malade, les esprits vitaux ont quitté notre corps. Le khmoc du père nous a frappé [par le biais de] 7 brāy qui nous font du mal; un cadavre se trouve enterré dans le sol. Modeler 1 image de Brah Laks dans un bè à 4 ruot [contenant] 6 kandon et le déposer au Sud. Guérison dans les 3 jours.»

15. doh dinamin nūv brah lāk kanāl mān kțīy aoy prayāt doḥ thkāt trūv kröttiyā gey dhvöh dos bvamn noḥ sasar drut dhvöh dos aoy sūn rūp taṃrīy 1 braḥ lāk 1 dūrabhīy 1 manussa boḥ ddhammm 1 beh rūtth 4 kandon் 4 jūn dov dāksīön thriaiy 3 sah hờ

«Si le siège est en Brạ Laks adulte: “...”22 S'il y a conflit, faire attention. Si l'on est malade, une personne qui nous veut du mal nous a jeté un sort; sinon, un pilier s'est creusé et nous porte malheur. Modeler 1 image d'éléphant, 1 de Brạ̣

22. Lacune dans le manuscrit (TK 307/B5: «Lorsqu'il est touché par la massue de Kumbhīlkār» [Rmk?]). 
Laks, 1 de Dhūbhī, 1 d'une personne à gros ventre [dans] un bè à 4 ruot [contenant] 4 kandon et le déposer au Sud. Guérison dans les 3 jours.»

16. doḥ dinamin nūv brạ̣ lāk cāss pān svoy rāj saṃmpātti dinaṃn் nūv nèh māan lābbh sabv prakāry" höy aoy prayāt pon் ph-Un En் srīy pruss nin dhvöh dos bvam noḥ jḥ̣h phdaiy ceñ jhām aṃbìy khlūn krè̀ trūv sahassa gey doh thkāt mān tammpūk bạ̣ noḥ mān anțùn bvam boḥ mān khmoc mèmutth dhvö dos aoy sūn rūp cor kān lambeñ 1 brān kān snāh 1 brạ̣ lāk 1 manuss brāy 3 beh rütth 7 kandon் 3 jün dov dāksīön thñaiy 3 sạ̣ hờ

«Si le siège est en Brah Laks vieillard: "Il jouit de la royauté" [Rmk ?]. Le siège étant celui-là, on a de la chance dans tous les domaines. Se défier des gens de notre famille, homme ou femme, qui nous veulent du mal; sinon, si l'on a mal au ventre et qu'on rejette du sang, quelqu'un nous a empoisonné. Si l'on est malade, c'est une termitière, sinon un puits, sinon un khmoc memat ${ }^{\prime 23}$ qui nous veut du mal. Modeler 1 image de brigand tenant une lance, 1 d'un chasseur tenant une arbalète, 1 de Brạ Laks, 3 de brāy [dans] un bè à 7 ruot [contenant] 3 kandoñ et le déposer au Sud. Guérison dans les 3 jours.»

\section{Rāb}

17. doh dinaṃn் nūv Rābbh kmīn pān svoy rāj saṃmpātti mān lābbh aotamm mān kț̄y anak ddhammm jūy doḥ thkāt put jhīy sec bvam noḥ mān gey cut nūv phdah noh aoy dos aoy sèn khmoc knan் phdah höy aoy sūn rūp brāy 4 beh rütth 4 kandon 7 jūn dov nīratīy thriaiy 3 sah hon

«Si le siège est en Rāb enfant: "Il jouit de la royauté" [Rmk?]. Chance extrême! En cas de conflit, une personne importante va nous aider. Si l'on est malade, on a mangé de la viande; sinon, c'est à cause d'un invité de passage dans la maison. Préparer un [plateau] d'offrandes à l'intention des khmoc. Modeler 4 images de brāy [dans] un bè à 4 ruot [contenant] 7 kanton et le déposer au Sud-Ouest. Guérison dans les 3 jours.»

18. [A29] doh dinaminn nūv Rābbh bāl bālī yak kandūy bāt aoy prayāt krèn strūv dhvöh dos nūv sabv thnaiy min sraṇuk citr doh thkāt brah bhūm aoy dos aoy sūn rūp brāy 7 beh rūtth 7 dun chāt bvöt jūn dov niratīy thriaiy 3 sah hon

«Si le siège est en Rāb adolescent: "Bālī l'entoure de sa queue" [Rmk 16]. Craindre qu'un ennemi nous veuille du mal. On a l'esprit sans cesse préoccupé. Si l'on est malade, c'est Brah Bhumi qui cause notre malheur. Modeler 7 images de $b r a \bar{y}$ [dans] un bè à 7 ruot [contenant] une bannière à étages et le déposer au SudOuest. Guérison dans les 3 jours.»

19. doh dinaṃm nūv rābbh kanṭāl pān setā mān lābbh höy niñ pan் vin put srīy

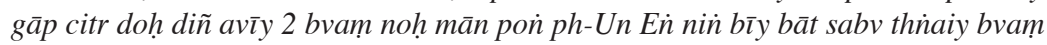

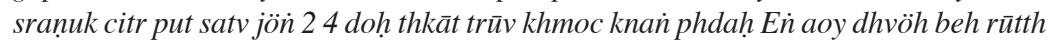
3 kandon 3 rūp brāy 1 chāt duñ paì dov nīratīy thriaiy 3 sah hon

23. memat': dieu du sol, génie protecteur ou familial. 
«Si le siège est en Rāb adulte: "Il détient Setā" [Rmk 18]. On a de la chance puis on la perd tout de suite, à cause d'une femme qu'on aime. Qu'on achète n'importe quoi, ou qu'il s'agisse de parents dans la difficulté, on a l'esprit sans cesse préoccupé à cause d'animaux bipèdes ou quadrupèdes. Si l'on est malade, c'est à cause d'un khmoc dans la maison. Faire un bè à 3 ruot [contenant] 3 kanton, 1 image de brāy, [une] bannière à étages et l'abandonner au Sud-Ouest. Guérison dans les 3 jours. »

20. doḥ dinaṃn nūv rābbh cāss trūv sar'brah rām bvam jā mān kț̄y cā̃̃ gey

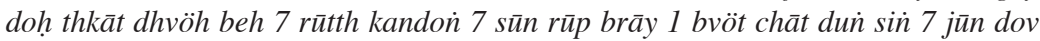
niratīy thñaiy 3 sạ hò

«Si le siège est en Rāb vieillard: "Il est touché par la flèche de Brạ̣ Raṃ” [Rmk 66]. Ce n'est pas bon. En cas de conflit, on perd. Si l'on est malade, faire un bè à 7 ruot [contenant] 7 kanton, 1 image modelée de brāy et 7 bannières à étages, et le déposer au Sud-Ouest. Guérison dans les 3 jours.»

\section{Nān Setā}

21. doḥ dinamin nūv setā kmīn mahā Īsīy yak jā cov dov ciñcīm duk khà gohā mān lābbh aotammm doḥ prus nin pān braḥbundh doḥ srīy nin bān phțīy bvam noh khñamm ratth gìmoh aoy pūjā brah groh nin mān lābbh doh thkāt trūv khmoc knaì phdah Ėं pa'pūl yak [A30] brāy mak dhvöh dos aoy dhvöh beh rūtth 3 kandon் 9 sūn rūp sīet nūv phkāh jhūk 1 yaks kān tammpon் 1 khmoc khvāk bhnek 1 pan dov puscim thriaiy 3 sah hon

«Si le siège est en Setā enfant: "Le Grand-ascète la prend pour petite-fille et l'élève dans la grotte" [Rmk 6]. Chance extrême! Un homme trouvera une épouse ; une femme, un mari. Sinon, un serviteur se sauvera. Préparer [un plateau] d'offrandes destiné au "siège de notre planète" ${ }^{24}$ pour retrouver la chance. Si l'on est malade, un khmoc dans la maison incite des brāy à venir nous faire du mal. Faire un bè à 3 ruot [contenant] 9 kanton, y placer dans un lotus 1 image de Yaks tenant un bâton et 1 de khmoc aveugle, et l'abandonner à l'Ouest. Guérison dans les 3 jours.»

22. doḥ dinaṃn nūv setā bāl rābbh lūc dov pān doḥ thkāt put bhit kantāl phlūv aoy prayāt krèn pāt drāby bvam noḥ krèn nūv cāk sṭān doḥ mān kț̄̄y aoy bịn anak ddhaṃm sammpor tāmmtè̀ kṭ̄y noh jhnah gey doḥ thkāt mamöh jḥ̣h kpāl trūv devatā ddhammm aoy dhvöh beh rūtth 3 kandon் 9 sūn rūp yaks kān tammpoì 4 pain dov puscim thriaiy 3 sah hon

«Si le siège est en Setā adolescente: "Rāb parvient à l'enlever" [Rmk 18]. Si l'on est malade, c'est parce que l'on a pris peur au beau milieu d'un chemin. Faire attention, on risque de perdre un bien. Sinon de partir de chez soi. En cas de conflit, s'en remettre à une personne importante de carnation cuivrée pour gagner. Si l'on est malade, [avec] délire et maux de tête, c'est un devatā puissant qui nous veut du mal. Faire un bè à 3 ruot [contenant] 9 kandori, 4 images de Yāks tenant un bâton, et l'abandonner à l'Ouest. Guérison dans les 3 jours.»

24. Brah groh : le «destin », ici le «siège » correspondant à l'âge du sujet, soit « Setā enfant». 


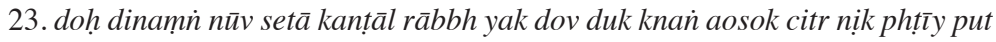
cāk sṭān doḥ thkāt lampāk ktāt pöh pvam thkāt löy krèn kūn cov ratth aoy prayāt krèn mān kțīy doḥ thkāt aoy dhvöh beh rūtth 3 kandon 9 sūn rūp sèh dim radeh 1 brāy 7 pan dov puscim thriaiy 3 sah hòn

«Si le siège est en Setā adulte: "Rāb l'enferme dans [le parc des] Asok" [Rmk 32]. Son cœur se morfond [à la pensée de] son mari qui a quitté le logis. Si l'on est malade, on souffre beaucoup; si l'on n'est pas malade, il faut craindre la fuite d'un enfant ou d'un petit-enfant. Faire attention. Craindre la survenue d'un conflit. Si l'on est malade, faire un bè à 3 ruot [contenant] 9 kandon, modeler 1 image de cheval attelé à une charrette, 7 de brāy, et l'abandonner à l'Ouest. Guérison dans les 3 jours.»

24. doh dinaṃn் nūv setā cāss brah rām vil dov vịn niñ pān drāby tọom cūl mak vịn doḥ dhvöh gun nin gey nin köt dos viñ hon doḥ thkāt aoy püjā braḥ groh höy yak cèñ krèn phṭ̂y brahbundh [bvam] gāpp [A31] citr gnāh doh thkāt aoy dhvöh beh

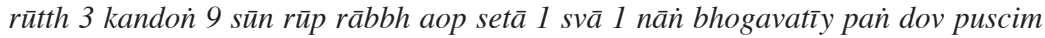
thriaiy 3 sah hon

«Si le siège est en Setā vieille: "Braḥ Rām revient [la délivrer]" [Rmk 67]. On rentre en possession d'un bien perdu. Si l'on aide quelqu'un, on en retirera du malheur. Si l'on est malade, préparer [un plateau] d'offrandes destiné au siège de notre planète et le sortir [de la maison] de peur que le mari et l'épouse se disputent. Si l'on est malade, faire un bè à 3 ruot [contenant] 9 kandoñ, modeler 1 image de Rāb étreignant Setā, 1 de singe, 1 de Bhogavatī et l'abandonner à l'Ouest. Guérison dans les 3 jours.»

\section{Setāyas}

25. doh dinaṃn் nūv setāyas kmīì braḥ rām yak jā klöh phan mān lābbh satv catupād dvepād doḥ thkät jhịh kpāl nīmit put gey cat'nūv phdah aoy dhvöh beh 4 rūtth kandon் 5 sūn rūp debtāh boh ddhaṃm 1 amröttiyūv 1 brāy 7 pan் dov bāyābv thriaiy 3 sah hon

«Si le siège est en Setāyas enfant: "Braḥ Rām en fait son ami” [Rmk 18,19]. Chance! Acquisition d'animaux quadrupèdes ou bipèdes. Si l'on est malade, qu' on a mal à la tête, c'est à cause d'une personne qui a séjourné dans la maison. Faire un bè à 4 ruot [contenant] 5 kandon, 1 image de devatā avec un gros ventre, 1 de Mrtyu, 7 de $b r \bar{a} y$, et l'abandonner au Nord-Ouest. Guérison dans les 3 jours.»

26. doh dinamin nūv Setāyas bāl sratīy khlāc tè ciñjīen brah daṃmruñ aoy prayāt māt niñ mān pöh pandos tpid māt noḥ doh thkāt thmah pāk bvam noh ciñjīen bvam snöt aoy dos mān pöh pan'min aoy paṇnan'aoy dhvöh beh rūtth 7 kandon் 9 sūn rūpp debtāh 7 pan் dov bāyābv thriaiy 3 sah hon

«Si le siège est en Setāyas adolescent: "Il dit craindre seulement l'auguste anneau" [Rmk 18]. Faire attention à ce qui sort de notre bouche. Sanctions encourues à cause de ce que l'on dit. Si l'on est malade, c'est une pierre cassée, sinon une bague [dont le gemme] a un défaut, qui provoque le malheur. [Sinon] c'est une promesse qu'on n'a pas tenue. Faire un bè à 7 ruot [contenant] 9 kandon், 7 images de devatā, et l'abandonner au Nord-Ouest. Guérison dans les 3 jours.» 
27. doh dinamin nūuv Setāyas kantāl pāk camm-Iñ krèn jhām ceñ aṃbīy khlūn kuṃ auy yak āsāh gey anak ddhaṃm dhvöh dos pan் khlūn bvam jā doh thkāt krèn mān ciñjīen bvam snöt auy dos bvam noh mān thmah pāk auy dos auy col pan auy dhvöh beh 3 rütth kandon 4 sūn rūp devatā ${ }^{*} k p a \bar{l} l 3$ taiy 6 pan dov bāyābv thriaiy 3 sah hon

«Si le siège est en Setāyas adulte: "Il se brise l'articulation de l'aile" [Rmk 19]. Craindre un écoulement de sang. Ne porter secours à personne [sous peine] d'être châtié par quelqu'un d'important. Malheur, mauvais! Si l'on est malade, c'est une bague défectueuse qui provoque le malheur; sinon c'est une pierre cassée. S'en débarrasser. Faire un bè à 3 ruot [contenant] 4 kantoñ, 1 image de devatā à 3 têtes et 6 bras, et l'abandonner au Nord-Ouest. Guérison dans les 3 jours.»

28. [A32] doḥ dinaṃn் nūv setāyas cāss braḥ rām lök khmoc nìn pan் drāby smöh pan் khlūn pāmmröh anak ddhaṃm auy prayāt rūc ambīy noḥ höy nin் mān lābbh hon் doh thkāt mān ciñjīen chār bvam noh krèn dov sṭhān E dīet mān pöh luss brah lịn tör min nūv knan் khlūn löy aoy sūn rūp manuss ddhāmm 1 brāy 8 beh rūtth 3 kandon் 8 pan் dov bāyābv thriaiy 3 sah hon

«Si le siège est en Setāyas vieillard: "Braḥ Rām enlève sa dépouille" [Rmk 19]. Perte de biens, comparable à la perte de la vie. [Si l'on] est au service d'une personne importante, faire attention. En dehors de cela, on a de la chance. Si l'on est malade, une bague s'est fendue. Sinon, nos esprits vitaux ont pris peur au cours d'un déplacement et ont quitté le corps. Modeler 1 image d'un être gros, de 8 brāy [dans] un bè à 3 ruot [contenant] 8 kandon், et l'abandonner au Nord-Ouest. Guérison dans les 3 jours.»

\section{Hanumān}

29. doḥ dinamin nūv hullamān kmī̀n brah ạdity nūv māt dinaṃn் nūv neh kuṃ ùt ān nin anak ddhamm löy krèn mān kṭ̄y aoy prayāt doh thkāt mān pöh chīy pamñöh gey phñöh bvam noḥ mān pöh chīy phlèh jhöh krahām bvạ̣ noḥ mān sasar phdaḥ mān bhin khāin E jön pöh gmān löy bhūm noh gey kāp sammlāp ghāh bīy mun aoy dhvöh beh rūtth 3 kandon 12 sūn rūp sèh 1 svā 1 brāy 6 pambun் srāh 1 pan dov autar thriaiy 3 sah hon

«Si le siège est en Hanumān enfant: "Le soleil est dans sa bouche" [Rmk 13]. Si le siège est celui-là, ne jamais se vanter devant une personne importante. Craindre un conflit; faire attention. Si l'on est malade, on a mangé quelque chose qu'on nous a offert, sinon on a mangé des fruits rouges, sinon c'est un défaut du pilier nord de la maison. Si ce n'est pas le cas, un crime y a été commis jadis. Faire un bè à 3 ruot [contenant] 12 kandon், 1 image de cheval, 1 de singe, 6 de brāy, 1 tube de bambou contenant de l'alcool, et l'abandonner au Nord. Guérison dans les 3 jours.»

30. doh dinamin nūv hullamān bāl brah rām prö dov kān stec mahā jammbūuv

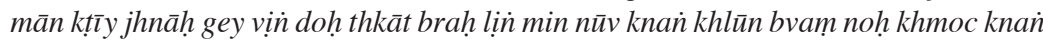

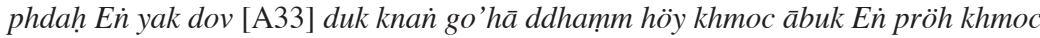
4 anak mak dhvöh dos kam aoy chīy cammnīy gey löy aoy dhvöh be rūtth 6 kandon் 6 sūn rūp debtāh dhaṃm 1 brāy 6 svā 1 pañ dov autar thriaiy 3 sah hò 
«Si le siège est en Hanumān adolescent: "Braḥ Rām l'envoie chez le roi Mahā Jamb" [Rmk 24]. En cas de conflit on gagne. Si l'on est malade, les esprits vitaux ont quitté le corps, sinon un khmoc dans la maison les a emmenés au fond d'une vaste grotte; [sinon] 4 khmoc envoyés par le khmoc de notre père nous font du mal. N'accepter aucun aliment de personne. Faire un bè à 6 ruot [contenant] 6 kandoñ, 6 images de grands devatā, 6 de brāy, 1 de singe, et l'abandonner au Nord. Guérison dans les 3 jours.»

31. doh dinamin nūv hullamān kanțāl kāl thvāy ciñjien braḥ dammrun் krèn trūv kțīy doh thkāt aoy dhvöh beh 6 rūtth kandon 6 sūn rūp Kippakār kān tammpon் mamonsak'1 buss 1 brāy 6 svah 1 pan dov aotar thinaiy 3 sah hon

«Si le siège est en Hanumān adulte: "Lorsqu'il va porter l'auguste anneau" [Rmk 30]. Craindre d'entrer en conflit [avec quelqu'un]. Si l'on est malade, faire un bè à 6 ruot [contenant] 6 kandoñ, 1 image de Kumpikar tenant une massue, 1 de serpent, 6 de $b r a \bar{y}, 1$ de singe, et l'abandonner au Nord. Guérison dans les 3 jours.»

32. doḥ dinamin nūv hullamān cāss dov tal lan̉kāh mak vịn mān kṭ̂̄y krèn trūv

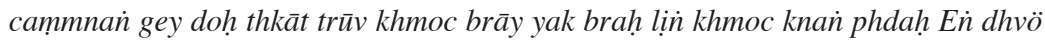
dos aoy dhvöh beh 6 rūtth kandon 6 sūn rūp debtāh ddhaṃm 1 brāy 6 prus 1 devatā kān sar'l pain dov aotar thinaiy 3 sạ hòn

«Si le siège est en Hanuman vieillard: "Il part à Lañkā et revient" [Rmk?]. En cas de conflit, craindre le sortilège de quelqu'un. Si l'on est malade, un khmoc-brāy a ravi nos esprits vitaux. C'est un khmoc dans la maison qui nous veut du mal. Faire un bè à 6 ruot [contenant] 6 kandon, 1 image de grand-devata, 6 de brāy, 1 d'homme, 1 de devatā tenant un arc, et l'abandonner au Nord. Guérison dans les 3 jours.»

\section{Les flèches de Braḥ Rām}

L'autre méthode de divination s'appuie sur la lecture de huit chiffres associés aux extrémités de quatre flèches entrecroisées disposées à l'intérieur d'un diagramme. Le Grū compte autant de chiffre que le sujet a d'années, revenant au chiffre 1 tout les neuf ans. Une série de huit chiffres disposés dans l'axe vertical du diagramme permet d'obtenir plus facilement le numéro de l'âge correspondant. Chaque flèche possède donc deux chiffres opposés : 1-8, 2-6, 3-7, 4-5, qui renvoient à deux épisodes de l'histoire de Braḥ Rām, celui de l'aileron indiquant toujours un augure favorable, celui du fer un augure défavorable. Dans ce dernier cas, il convient d'opérer un rite d'expulsion du mal avec confection d'un bè spécial en offrande à Braḥ Rām.

Quatre doubles formules sont associées aux quatre flèches : 


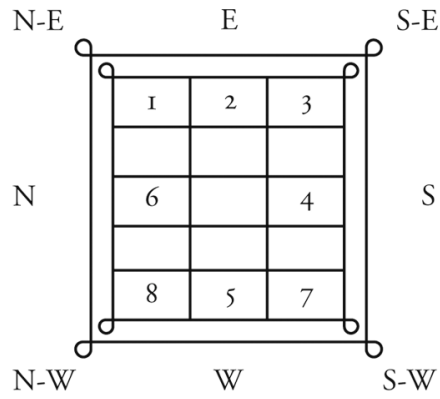

Fig. 3

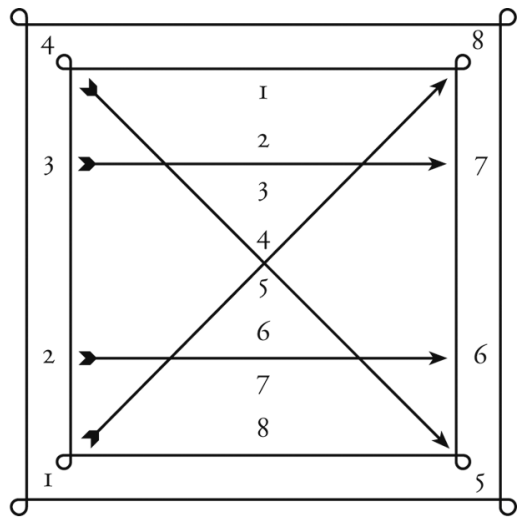

Fig. 4

Les quatre flèches.

Flèche 4-5

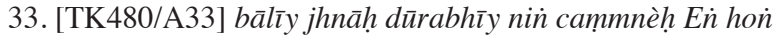

«Bāli vainc Dūbhī par son propre savoir.» [Rmk 20]

34. setāyas trūv brah dammrruñ pāk caṃ-Iñ' slāp bvam jā

«Setāyas est touché par l'auguste anneau qui brise l'articulation de son aile.» [Rmk 19] Mauvais!

Flèche 3-7

35. dūrabhīy prayut nin bālīy [A34] pān sammmpātti ābuk jā

«Dūbhī se bat avec Bāli et obtient les richesses de son père.» [Rmk 20] Excellent!

36. rābbh trūv sar brah rām pañ setāh bvam jā aoy krèn

«Rāb reçoit la flèche de Brạ̣ Rām qui retrouve Setā.» [Rmk 66] Mauvais! Se méfier!

Flèche 2-6

37. braḥ rām thlèn sar trūv rābbh pān setā vĩ̃ jā braḥbundh

«Braḥ Rām tire une flèche sur Rāb et retrouve Setā son épouse.» [Rmk 66]

38. bālīy cā̃̃ dūrabhīy aoy krèn amböh gey bvam jā

«Bālī perd devant Dūbhī.» [Rmk 20] Se méfier des sortilèges. Mauvais!

Flèche 1-8

39. brah rām vāt kovdān pā̃n rābbh jā begy

«Brah Rām bande son arc et touche Rāb.» [Rmk 66] Excellent!

40. dūrabhīy trūv brah khān bālīy slāp bvam jā aoy krèn

«Dūbhī est touché à mort par l'épée de Bālī.» [Rmk 20] Mauvais! Se méfier !

Ces épisodes ne sont cependant pas tous évoqués de façon identique et l'on compte un certain nombre de variantes, que voici : 
41. [TK307/B1] doh dịnāmin nau brah rām kmịn lok jrom rāsār nau nī̀n sammpāt debtār caṃön oy jaiy tpītth brạ̣ rām prāp "sar trūv kā phdaiy krom

«Si le siège est en Braḥ Rām enfant: "Le seigneur protège les biens des dieux, les fait prospérer vers la victoire, car Braḥ Rām chasse les ennemis pour sauver le monde d'en bas".» [Rmk 7]

42. [TK307/B6] doh dịnāmin nau rāp' bāl' kāl [B7] dauv luoc brahbun brah ind kāl cuḥ ceñ mak "kakrök dāmñ phdaiy kraum

«Si le siège est en Rāb adolescent: "Lorsqu'il abuse de l'épouse de Brạ̣ Indr. Quand il ressort (du palais), le monde d'en bas tremble".» [Rmk 6]

43. [TK307/B8] doḥ dịnāminn nau setārr kmin stec duk knà் bon் bāy pan stav' ddau nār chner sdīn mahār r rssī ghöñ anak rös yak jā cauv nāmin dau āsrammmm

«Si le siège est en Setā enfant: "Le roi la place sur un radeau qu'il abandonne au fil de la rivière. Le Grand-ascète la voit, la recueille, l'adopte comme sa petite-fille, la ramène à l'ashram".» [Rmk 6]

44. [TK307/B2] doḥ dịnāmin nau kha kmịn mụāy aup rāksārr jā stec

«Si le siège est en Khar enfant: "Sa mère le protège avec affection ; il est roi". » [Rmk 20]

45. [TK307/B2] doḥ dịnāminn nau kha bāl' pān dau rien haur

«Si le siège est en Khar adolescent: "Il va apprendre la divination".» [Rmk?]

46. [TK307/B2] doh dịnāminn nau kha kandạl kāl dau cpāin nìn brah lākkh' trūv "sar

«Si le siège est en Khar adulte : "Lorsqu'il combat Brạ̣ Laks et reçoit la flèche"» [Rmk?].

47. [TK307/B3] doḥ dịnāminn nau khar cās prās rājj saṃmpātt'

«Si le siège est en Khar vieillard: "Il perd la royauté".» [Rmk?]

48. [TK307/B3] doḥ dịnāmn் nau brah lākkh' khmī̀n mọāy rāksār thnam

«Si le siège est en Brah Laks enfant: "Sa mère le protège".» [Rmk?]

49. [TK307/B13] doḥ dịnāminn nau hunnamānd' kmịn braḥ āditth krạ̣ek chèh pān slāp brah aditth möl mak yal' tūc svā 1 brah ạdit prasap' ras' lön hu"nmān mān rițti anubhāpp mān brah āddit dāmin 7 knaì mātth

«Si le siège est en Hanumān enfant: "Braḥ Adity le brûle d'un coup d'œil; il meurt. Brah Adity regarde et aperçoit comme un singe; il le ressuscite. Hanumān [en] obtient une grande puissance; il a sept soleils dans la bouche".» [Rmk 13]

50. [TK307/B4] doḥ dịnāmn் nau brah lākkh bāl kāl töh des tām brah rāmm sdec le lā prāp chner sdön tāp kūn devatār dāmin 7 anak lök dhnuor sil anak tār mahā $\bar{R}$ ssịy ön 2 chchau 2 brah rām sḍec E brah rāmm pröh brah lākkh cūl dau möl key ghö̃̃ key lök dhnuor sil anak tā mahār $\bar{R}$ ssịy thā pöh anak eñār lök dhnuoh neh rūc nịn oy cau añ jā prabun neh döbd brạ läkk vil mak krāp duol brah rāmm ththā key lökkh dhnuor sil mahār R̦ssiy pö anak enāar lök ruoj nịn oy cauv jā brahbun'

«Si le siège est en Brah Laks adolescent: Lorsque Brah Rām quitte la ville, Brah Laks le suit. Ils cheminent le long des rivières et croisent sept fils de dieux [qui vont] soulever l'arc magique du vénérable Grand-ascète: eug! eug! chau ! chau! Braḥ Rām entend [le tumulte de l'épreuve] et envoie [Brah Laks] observer [les concurrents]. Le vénérable Grand-ascète dit: "À celui qui soulèvera cet arc, je 
donnerai ma petite-fille pour épouse". Brah Laks revient, se prosterne devant Brah Rām et dit: "Ils soulèvent l'arc magique du Grand-ascète; à celui qui y parvient il donnera sa petite-fille pour épouse".» [Rmk 14].

51. [TK307/B9] doḥ dịnāmin nau setā bāl' brah rāmm lök dhnūr sil bhnāl pān sètārr jā prabun' brah rāmn nāmm sètār bāk kanḍāl phluov tāp sūr panakkhā

«Si le siège est en Setā adolescente: "Braḥ Rām concourt pour soulever l'arc magique et obtient Setā pour épouse. Il l'emmène. À moitié chemin, ils rencontrent Sūrpanakhā".» [Rmk?]

52. [TK307/B6] doḥ dịnāmin nau rāp kmī̀n kāl dau vamahorr srapp'pradāh nìn bāl̄̄y gāt ththā jā kdam'gron் gāt yak mak can oy sūrgrip lèn

«Si le siège est en Rāb enfant: "Lorsqu'il va au spectacle et tombe sur Bālī, celui-ci dit: Rāb s'est transformé en gros crabe de rizière! Il l'attrape, le ficelle et le donne pour que Sugrīb s'amuse".» [Rmk 17]

53. [TK307/B10] doḥ dịnāminn nau catāyuḥ kmī̀n mụāy aup rāksā

«Si le siège est en Setāyas'enfant: "Sa mère le protège avec affection".» [Rmk?]

54. [TK307/B11] doḥ dịnāmñ nau catāyuh bāl kāl dauv rien sil nịn brah isūr brah nar rāy brah dhan̄y phan oy phlās khsèr sèmā brātth lok noh döbv brah isur anak srāy lèn dauv

«Si le siège est en Setāyas'adolescent: "Lorsqu'il va apprendre la magie auprès de Braḥ Isūr, Braḥ Narāy, Braḥ Dharaṇī. Il remplace les sīmā en lanières [de peau de buffle] du séjour, afin que Brah Isūr parvienne à les défaire”.» [Rmk?]

55. [TK307/B11] kāl cattarryuḥ dau yal rāpp namm sètārr catāryuh kāñ cum in

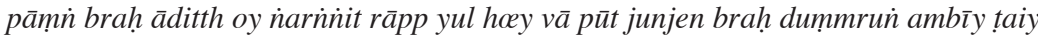
nā̄n setār ccaul dauv trūv pāk cạ̣ in catāyuh ṭ̣it yak āsār braḥ rāmm

«Lorsque Setāyas voit Rāb emporter Setā, il étend les ailes, voile le soleil, provoque l'obscurité. Voyant cela, Rāb arrache l'auguste anneau du doigt de Nān Setā et le lance, brisant l'articulation de Setāyās parce que celui-ci porte aide à Braḥ Rām.» [Rmk 18]

56. [TK307/B12] doḥ dịnāṃn் nau catāyuh kantāl'braḥ rām yak dau moel bvaṃ mān sah locy

«Si le siège est en Setāyas adulte: "Braḥ Rām emmène Setāyas pour le soigner. Il ne guérit pas".» [Rmk 19]

57. [TK307/B12] doḥ dịnāminn nau catāyuh cās kāl slāp'dauv koe jā grudhdh rājj mān ammmāj jā ammcāss is anak jā mitth nin brah rāmm tèn juoy kār phdaiy kraum

«Si le siège est en Setāyas vieillard: "Lorsqu'il meurt, il renaît en roi des Garuda, puissant, et maître de tout le monde. Il est l'ami de Brạ̣ Rām et l'aide à sauver le monde d'en bas".» [Rmk 19]

58. [TK307/B14] doh dịnāmin nau hunamm ān kandal kāl brặ rām proh dau yak s"ar ambīy pād bhnamm brah sūmèh hoh jrèk sdịn [B15] 1 doebv pān s"ar noh

«Si le siège est en Hanumān adulte: "Lorsque Braḥ Rām l'envoie se procurer la flèche au pied du Mont Sumer; il s'envole, plonge dans la rivière, et l'obtient".» [Rmk?]

59. [TK307/B7] doḥ dịnāminn nau Rāpp kandāl kāl pañ bīybhèkkh oy pok klas'jaiy brah rāmmm thlèn s"ar nèh tec ratn kumbal 
«Si le siège est en Rāb adulte: "Lorsque l'aîné Bibhek fait ouvrir le parasol de victoire; Braḥ Rām tire une flèche et déchire le tissu précieux”.» [Rmk 36]

60. [TK307/B5] doḥ dịnāmin nau brạ̣ lākkh'kanḍāl'kāl trūv mon்kasāks gummburkār

«Si le siège est en Brah Laks adulte: "Lorsqu'il est touché par la massue de Kumbhīkar". » [Rmc 38]

61. [TK307/B8] doḥ dịnāminn nau braḥ rām thlèn sar trūv thlœm slāp pan் lun்kā

«Si le siège est en Rāb: "Braḥ Rām lui décoche une flèche dans le foie; il meurt et perd Lankā".» [Rmk?]

62. [TK307/B1] doḥ dịnāmin nau braḥ rām cāss pān soy rājj saṃmpāt jā cau braḥ nagar is srīy snaṃm krammakār ddebvī ddhạ̣ tūc brạ̣ rāmm loxi jā sḍec kāl sètā gūv rūp rāp ou srīy snam [kramma] kāri moel

«Si le siège est en Braḥ Rām vieillard: "Il jouit de la royauté, maître de l'auguste cité, de toutes les servantes, épouses et concubines; il devient roi. Lorsque Setā dessine le portrait de Rāb pour le faire voir aux servantes".» [Rmk 72]

63. [TK307/B9] doh dịnāmin nau sètā kandal kāl braḥ rām pān mak oy jā debīy ddhammm brah rām bvam duk cit sètā loey

«Si le siège est en Setā adulte: "Lorsque Brah Rām la retrouve, il en fait sa première épouse. Il n'a pas confiance en elle".» [Rmk 72]

64. [TK307/B10] doḥ dịnāṃn் nau sètār cās mān 1 jā 2 nìn braḥ rāmm bvam pān loy sūv slāp khluon

«Si le siège est en Setā vieille : "Les soupçons de Braḥ Rām lui sont insupportables; elle préfère mourir".» [Rmk 72]

65. [TK287/B20] kāl rāb prèh kāy jā brāhm briddhā

«Lorsque Rāb prend l'apparence d'un vieux brāhmane.» [Rmk 16]

66. [TK287/B3] brah lakkh ānucjā stec drañ cehstār toy nūv vitththīy kan'là

«Brah Laks: "Le cadet possède le pouvoir grâce à la voie correcte".» [Rmk 12]

67. [TK287/B3] kāl sèr [B4] tā nīmul rāb vā dhvœr kal dauv duk knañ tom ausauk

«Lorsque Rāb abuse de la pure Setā et qu'il va la déposer au Parc des Asok.» [Rmk 32]

68. [TK287/B3] brah rāmm stec tām sèrtār lampāk bruoy prān moḥh̄mmār

«Brah Rām poursuit Setā et éprouve beaucoup de peine.» [Rmk 22]

69. [TK287/B4] bīybhek bunrāy drà் rīdhīy kāy bāllār aṇnāj

«Bibhek, le resplendissant, possède un grand pouvoir.» [Rmk ?]

70. [TK287/B4] kran் rabd bhanār cīt drœes mahhīmmār pamphlāñ luñkār

«Le roi Rāb au cœur sans aucune pitié fait détruire Lañkā.» [Rmk?]

71. [TK307/45] dūbhīy tor braiy kum ūt à ātmāśs è

«Dūbhī marche en forêt: Ne pas se vanter soi-même.» [Rmk 20]

\section{L'horoscope perdu des devins}

Ces soixante et onze extraits s'ordonnent ainsi pour nous livrer les grandes lignes d'une version du cycle que nous ne connaissons pas au Cambodge. 
J'ai reconstitué leur enchaînement (de 1. à 35.) en suivant grosso modo le déroulement habituel de l'histoire ${ }^{25}$, telle qu'elle se présente partout. Les numéros donnés entre parenthèses renvoient aux paragraphes traduits ci-dessus.

1. Rāb jouit de la royauté (§ 17) et règne sur Lañkā (§ 61).

2. Brah Rām prend naissance pour sauver le monde d'en bas (§ 41$)$.

3. Rāb s'introduit dans le palais de Brah Indr et abuse de la femme du dieu $(\S 20,42)$.

4.Pour se venger, celle-ci renaît dans le sein de l'épouse de Rāb, lequel abandonne le nouveau-né sur un radeau $(§ 43)$.

5. Elle est recueillie par le Grand-ascète qui l'élève dans la grotte de l'ermitage $(\S 21,43)$.

6. Hanumān prend naissance. Brạ Adity le brûle d'un coup d'œil mais le ressuscite. De ses mésaventures avec le soleil il acquiert une grande puissance $(\S 29,49)$.

7. Départ de Brah Rām, suivi de Brạ Laks. Leur rencontre avec les dieux qui s'essayent à soulever l'arc magique (\$50).

8. Brah Laks est envoyé en reconnaissance et aperçoit Nān Setā dans la grotte (§13).

9. Braḥ Rām emporte la victoire et se marie avec Nān Setā $(\S 5,6,51)$.

10. Le sage Brah Laks pratique les observances ( $\$ 14)$; il possède le pouvoir grâce à la voie correcte ( $\$ 66)$.

11. La rencontre de Braḥ Rām avec Sūrpaṇakhā (§ 51).

12. Khar est roi et sa mère le protège (\$ 44). Il va apprendre la divination (\$ 45). Lorsqu'il combat Brah Laks il reçoit la flèche (§ 46) et perd la royauté (§ 47).

13. Bālī s'empare de Rāb à l'aide de sa queue (§16).

14. Rāb se métamorphose en crabe mais, découvert par Bālī, il est offert comme jouet à Sugrīb (\$ 52).

15. Naissance de Setāyas, sa mère le protège ( $(53)$. Il s'éduque dans les sciences magiques auprès de Brah Isūr, Braḥ Narāy, Brah Dharan̄ī et remplace les fils de sīmā afin que Brah Isūr les défasse (§ 54). Il devient l'ami de Braḥ Rām $(\S 25,57)$.

16. Épisode de la gazelle d'or ( $\$ 7)$.

17. Rāb prend l'apparence d'un vieux brahmane et enlève Nāñ Setā $(\S 6,22,65)$.

18. Setāyas intercepte Rāb, étend les ailes, voile le soleil, provoque l'obscurité. Rāb lui brise l'articulation en lui jetant la bague de Nān Setā (§ 34.55). Il est terrassé suite à l'imprudence de ses propres paroles (§ 26-28).

19. Braḥ Rām ne parvient pas à guérir les blessures de Setāyas (§ 56) qui renaît alors en roi des Garuda et l'aide à sauver le monde d'en bas ( $\$ 29,57)$. Rāb dépose Nān Setā dans le Parc des Asok $(\S 19,23,67)$.

22. Naissance secrète de Dūbhī qui terrasse $(\S 9,35)$ et obtient les richesses de son père $(\S 10)$, puis s'en va orgueilleusement défier Bālì à l'intérieur d'une grotte, où il trouve la mort en vertu du savoir de son adversaire $(\S 11,12,33,38,40,71)$.

25. Cf. traduction française: Bizot 1989. 
23. Braḥ Rām part à la recherche de Nān Setā et en éprouve beaucoup de peine (§68).

24. Bibhek est vice-roi de Lañkā (§1), ses pouvoirs sont merveilleux (§ 69). Il annonce une catastrophe si Rāb ne libère pas Nān Setā et Rāb le bannit (§ 2).

25. Hanumān, au service de Brạ̣ Rām, est envoyé chez Mahājambū (§ 30).

26. Il s'envole ensuite à Lañkā afin de remettre à Nān Setā un anneau de la part de Braḥ Rām $(\S 31,32)$.

27. Braḥ Rām l'envoie se procurer une flèche au pied du Mont Sumer (§ 58).

27. Rāb ouvre le parasol de victoire mais Brạ̣ Rām le déchire d'une flèche ( $\$ 59)$.

28. Braḥ Laks est blessé par la massue de Kumbhīkar (\$60).

29. Braḥ Rām tue finalement Rāb à l'aide d'un trait $(\S 20,39,61)$, il est sacré roi (§ 62) et retrouve Nān Setā $(§ 24,36,37)$.

30. Il fait de Nān Setā sa première épouse, mais n'a pas confiance en elle (§63).

31. Nān Setā dessine le portrait de Rāb (§ 62).

32. Les accusations de Braḥ Rām lui sont insuportables (§63), elle préfère mourir (§ 64).

33. Braḥ Rām fait régner Bibhek à Lañkā (§ 4).

34. Bibhek et Brah Laks règnent en paix $(\$ 4,16)$.

35. Braḥ Rām jouit des richesses de Lankāa $(\S 8,61)$.

Dans la brochure que la «Commission du Ramker» a publiée à Phnom Penh en 1969, il est brièvement rapporté l'existence d'une histoire ésotérique des aventures de Brạ̣ Rām, gardée secrètement, sous une forme qui «rappelle la Bible, et offre, comme elle, des scènes allégoriques servant à prédire l'avenir ${ }^{26}$. En réalité, l'histoire en question ne se réfère à aucune sorte d'enseignement occulte; c'est justement ce qui la caractérise et par quoi elle se différencie.

Il s'agit au contraire d'une version spéciale dédiée aux vaticinations des devins, dissimulée en tant que telle derrière leurs prophéties et disparue de la scène parce que l'imaginaire ne pouvait se la représenter que par bribes, jamais la saisir dans la continuité de ses parties. De ce fait, elle nous est parvenue émiettée mais sans doute inchangée, sa transmission au Cambodge par formules cloisonnées lui permettant d'échapper au risque de sa refonte dans le modèle unique. La composition se rapproche de celle du Rāmāyaṇa de Vālmīki ${ }^{27}$, et de ses variantes en Inde et en $\mathrm{Asie}^{28}$, alors même que le détail des scènes diverge assez peu de celles du Rāmaker ${ }^{29}$, sauf là où se concentrent les interpolations qui lui confèrent une portée gnostique.

26. Hang Tung Hak $1969: 26$.

27. Cf. Roussel 1903. Hari Prasad Shastri 1957, 1959, 1962.

28. Raghavan 1975, 1980. Iyengar 1981. Bizot 1989: 26-29.

29. Ou de manière peu significative : Khar est curieusement instruit dans les sciences divinatoires, il reçoit une flèche de Brạ̣ Laks et non de Brạ̣ Rām (8-9); Rāb métamorphosé en crabe est donné comme jouet à Sugrīb et non comme esclave à 
Précisément, les astrologues nous disent que le seul vainqueur, le véritable héros, est resté comme en Inde celui qui tue Rāb, autrement dit Braḥ Rām, et non pas le Singe blanc Hanumān («Fils du vent», i.e. le «souffle» du méditant) ${ }^{30}$. Ainsi, quand le Rāmaker porte l'accent sur la grandeur de Brah Rām qui est d'ordre spirituel et sur sa «gloire» que symbolise l'union avec Setā, ceux-ci se contentent d'octroyer les biens terrestres au vainqueur et n'hésitent pas le moins du monde à faire disparaître Nān Setā. Hanumān: son rôle s'achève à son retour de Lañkā; Setā: son destin est néfaste, elle décide de mourir; Brah Rām: il vit seul au terme de son existence. Les deux époux ne se retrouvent donc pas et il n'est fait aucune mention à la cérémonie funèbre, alors que pour les Khmers la mort de Brạ Rām est l'épisode obligé de la fin du récit, parce que c'est elle qui préfigure les rites de la dissolution du composé physique avant la jouissance de l'immortalité ${ }^{31}$.

Aucun échafaudage intellectuel ou religieux n'a donc eu prise sur cette composition rigide, intangible, dont le sort des protagonistes ne pouvait être que la préfiguration des destinées humaines. Quelle que soit la forme originale sous laquelle cette version fut introduite au Cambodge - je veux dire dans son rapport avec l'astrologie indienne -, les devins nous l'ont transmise telle quelle, avec ses deux procédés (les huit influences et les flèches de Rām), en prenant soin de ne pas la convertir au cycle chronographique des douze animaux inconnus en Inde, et encore moins de l'illustrer à l'aune de leurs propres idées. La culture indienne avait si parfaitement modelé la pensée qu'une référence à Rāb ou à Setāyas, non seulement suffisait à exprimer le sacré d'une situation donnée, mais encore l'idéalisait en la rendant exemplaire et fournissait la solution ou l'explication requise pour déchiffrer l'oracle.

Angat (21); Setāyas est instruit dans les sciences magiques au cours de circonstances qu'on ne situe pas (23); Hanumān procure une flèche à Braḥ Rām qu'il va chercher au pied du Mont Sumer, à la suite d'événements qu'on ne connaît pas (50); Rāb ouvre le parasol de victoire sur le conseil de Bibhek (51) alors que celui-ci est déjà censé être passé à Braḥ Rām; Braḥ Laks est blessé par la massue de Kumbhīkār (52).

30. Bizot 1989: 29.

31. Bizot 1989: 42-61. 


\section{SOURCES MANUSCRITES}

TK 280: Ta Sun, Phum Krang Thnung, Khum Krang Thnung, Srok Phnom Penh, Khèt Kandal, Cambodge.

TK 287: Krou Em, Vat Phnom Thom, Srok Mongkol Borey, Khèt Battambang, Cambodge.

TK 300 : Achar Chum, Phum Prey Sala, Khum Krang Thnung, Srok Phnom Penh, Khèt Kandal, Cambodge.

TK 307: Krou Trok Din, Phum Samrong Teav, Khum Krang Thnung, Srok Phnom Penh, Khèt Kandal, Cambodge.

TK 480: Achar Rit, Ban Ta Tiou, Ampeu Prasat, Tambon Surin, Changvat Surin, Thaillande.

\section{Bibliographie}

BILLARD, Roger (1963). «Les cycles chronographiques chinois dans les inscriptions thaïes». Paris, Bulletin de l'École française d'Extrême-Orient. Vol. 51. Num. 51-2: 403-431.

Bızot, François (1973). Histoire du Reamker. Rīöñ rāmakerti nai tā cak'. Récit recueilli et présenté par-. Phnom Penh (Seconde édition, Bangkok 1980).

Bızot, François (1976). Le Figuier à cinq branches. Recherches sur le bouddhisme khmer, I. Paris, Publication de l'École française d'Extrême-Orient, vol. CVII.

Bızot, François (1981). «The Reamker». Asian Variations in Rāmayāna. Paper presented at the International Seminar on "Variations in Ramāyāna in Asia: Their Cultural, Social and Anthropological Significance ». K. R. Srinivasa Iyengar (dir.). New Delhi, Sahitya Akademi: 262-275.

Bızot, François (1989). Rāmaker ou l'amour symbolique de Rām et Setā, Recherches sur le bouddhisme khmer, V. Paris, Publication de l'École française d'ExtrêmeOrient, vol. CLV.

Bızot, François (1992 a). Le Chemin de Laìkā. Textes bouddhiques du Cambodge, I. Chiang Mai-Phnom Penh, Publication du Fonds pour l'édition des manuscrits, École française d'Extrême-Orient.

Bızot, François (1992 b). «Le dhammakāya, corps de Bouddha ». Le Chemin de Laìkā. Textes bouddhiques du Cambodge I. Chiang Mai-Phnom Penh, Publication du Fonds pour l'édition des manuscrits, École française d'Extrême-Orient: 293-300.

Bızot, François (1993). Le Bouddhisme des Thaïs. Brève histoire de ses mouvements et de ses idées des origines à nos jours. Bangkok, Édition des Cahiers de France.

Bızot, François (1996). La Pureté par les mots. Textes bouddhiques du Laos, I. ParisChiang Mai-Phnom Penh-Vientiane, Publication du Fonds pour l'édition des manuscrits, École française d'Extrême-Orient.

Bızot, François (1998). «Bouddhisme, Asie du Sud-Est». Dictionnaire critique de l'ésotérisme. Jean Servier (dir.). Paris, PUF: 221-231. 
Bızot, François (2000). «La place des communautés du Nord-Laos dans l'histoire du bouddhisme d'Asie du Sud-Est ». Paris, Bulletin de l'École française d'ExtrêmeOrient, Mélange du centenaire, t. 87-2: 511-528.

CÆEDÈs, George (1934). «L'origine du cycle des douze animaux au Cambodge». T'oung Pao, Archives pour servire à l'étude de l'histoire, des langues, de la géographie et de l'ethnographie de l'Asie orientale. Vol. 31, livr. 3-5. Leide, E. J. Brill: 315329.

Finot, Louis (1917). «Littérature laotienne». Paris, Bulletin de l'École française d'Extrême-Orient: 76-83.

GuENZI, Caterina (2010). Anthropologie de la divination dans le monde indien. Séminaire 2010-2011 - Compte rendu. Paris, École des Hautes Études en Sciences Sociales.

HANG Thun Hak (dir.) (1969). Ramker ou Ramayana khmer. Commission du Ramker. Université Royale des Beaux-Arts. Phnom Penh, Sangkum Reastr Niyum.

HARI Prasad Shastri (trans.) $(1957,1959,1962)$. The Ramayana of Valmiki. Londres, Shanti Sadan.

IYENGAR, K. R. Srinivasa (dir.) (1981). Asian Variations in Rāmayāna. Paper presented at the International Seminar on "Variations in Ramāyañna in Asia: Their Cultural, Social and Anthropological Significance». New Delhi, Sahitya Akademi.

MartinI, François (trad.) (1978). La Gloire de Rāma. Rāmakerti. Rāmāyaṇa Cambodgien. Introduction et notes de Ginette Martini. Préface de Solange Thierry. Paris, Les Belles Lettres.

LeCLÈre, Adhémard (1909). «Le Zodiaque Cambodgien». Revue des études ethnographiques et sociologiques. Arnold van Gennep (dir.). Paris, juillet-août, Paul Geuthner: 159-174.

Nicolas, René (1928). «Le Ramayana Siamois». Extrême-Asie - Revue indochinoise. Hanoi, Imprimerie d'Extrême-Orient. № 19, janvier : 297-308. № 21, mars : 409422. $\mathrm{N}^{\circ} 23$, mai: $565-580$. $\mathrm{N}^{\circ} 25$, juillet: 22-31.

Porée-Maspero, Éveline (1962). «Le cycle des douze animaux dans la vie des Cambodgiens». Paris, Bulletin de l'École française d'Extrême-Orient, t. L, fasc. 2: 311-325.

RAGHAVAN, Venkataraman (1975). The Ramayana in Greater India. Surat, South Gujarat University.

RaghaVAN, Venkataraman (1980). The Ramayana tradition in Asia. New Delhi, Sahitya Akademi.

Roussel, Alfred (trad.) (1903). Râmâyana de Vâlmîki. Paris, Maisonneuve Frères, «Bibliothèque orientale», vol. VI, VII, VIII.

Wales, H.G. Quaritch (1983). Divination in Thailande, The hopes and fears of a Southeast Asian people. Londres et Dublin, Curzon Press. 
François Bizot

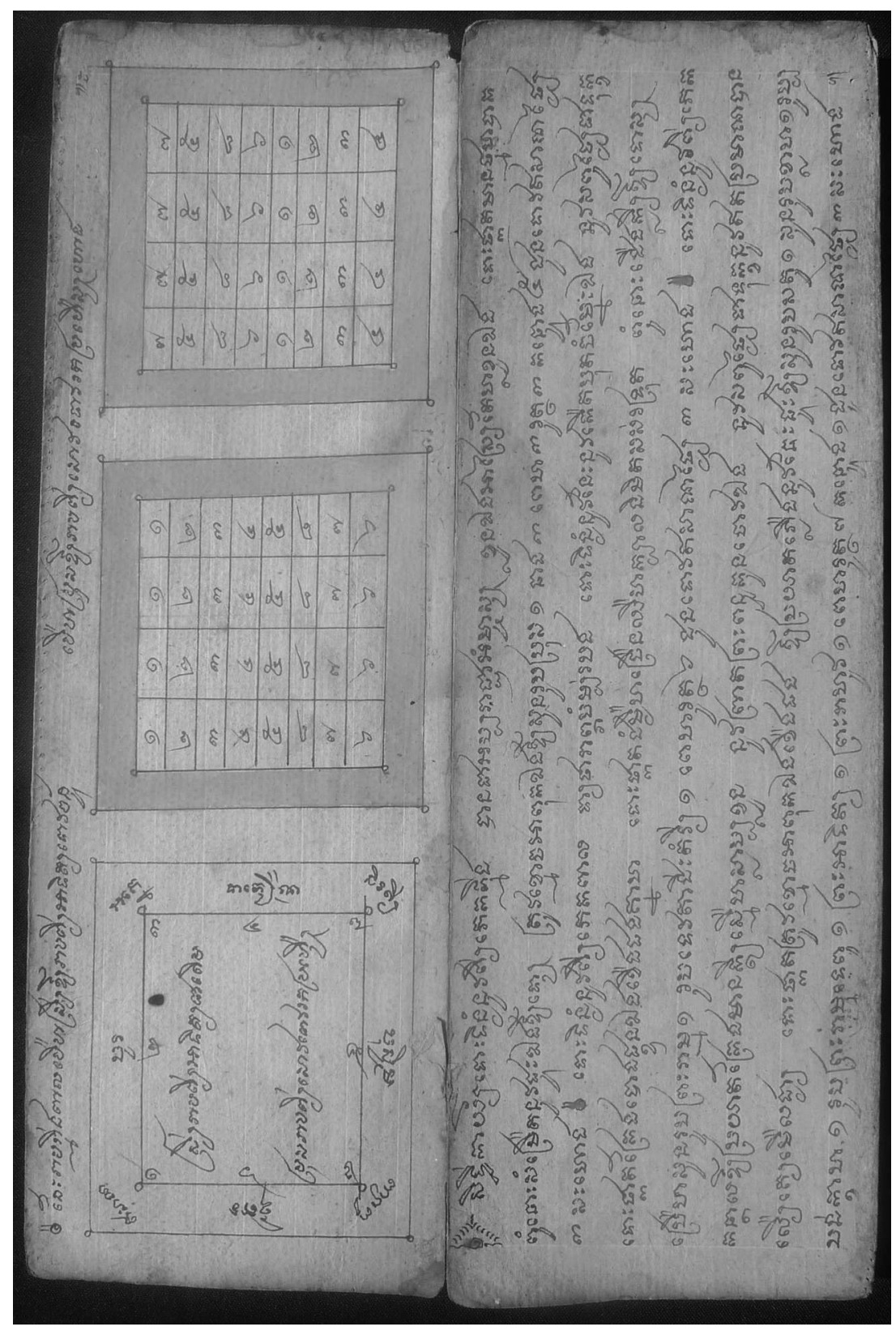

TK 480, feuillet A23. 


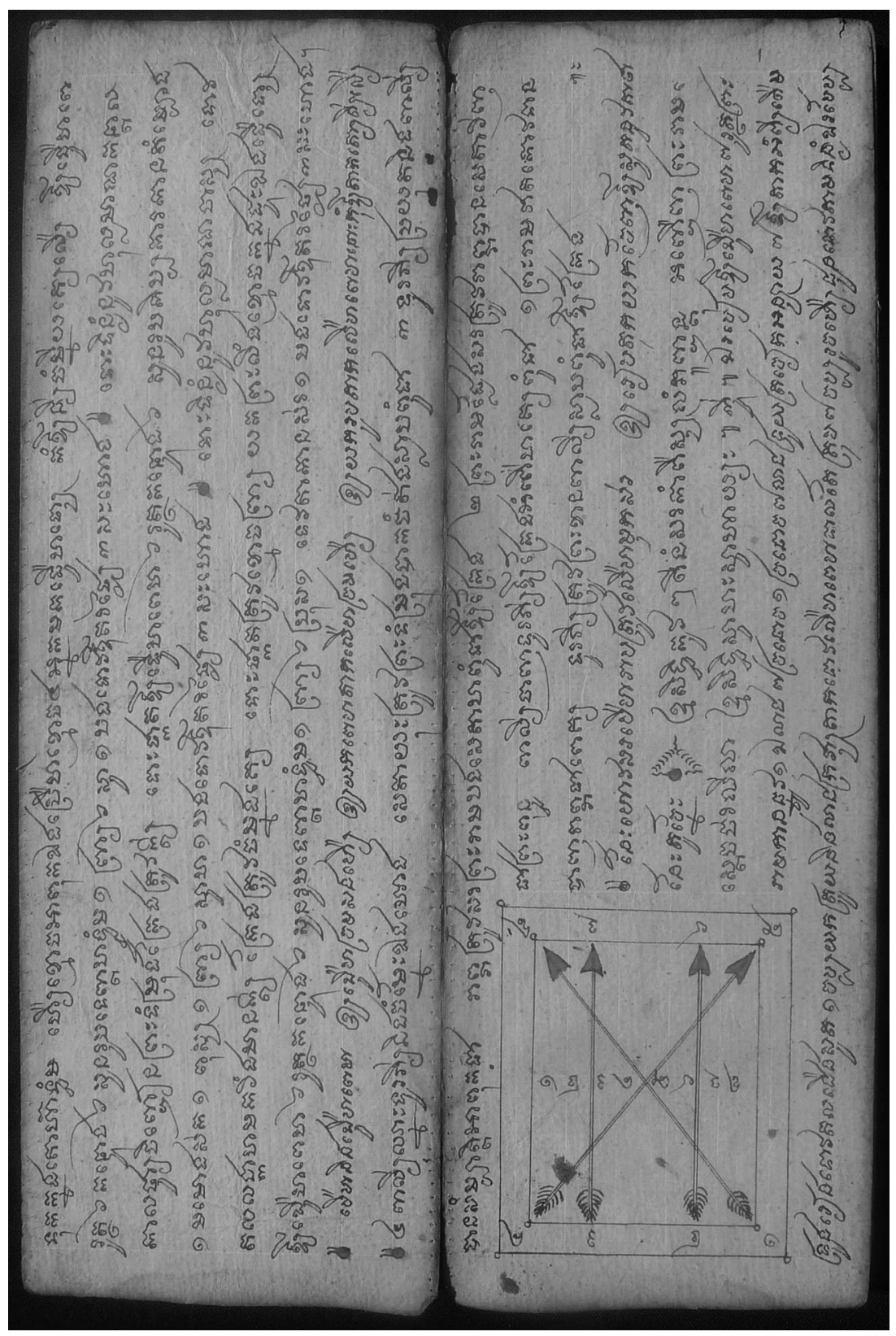

TK 480, feuillet A33. 
